\title{
Low quantum efficiency of $\mu$-oxo iron bisporphyrin photoacatalysts explained with femtosecond M-edge XANES
}

Received 00th January 20xx, Accepted 00th January 20xx

\author{
Kori Sye, ${ }^{{ }^{\dagger}}$ Clare Leahy, ${ }^{a^{\dagger}}$ and Josh Vura-Weis ${ }^{a^{*}}$
}

DOI: $10.1039 / x 0 x \times 00000 x$

Bridged $\mu$-oxo iron bisporphyrins serve as photocatalysts for oxidative organic transformations, but suffer from low quantum efficiency. We use femtosecond optical and $M_{2,3}$-edge XANES spectroscopy to investigate the early photodynamics of the $\mu$-oxo iron bisporphyrin, (TPPFe) ${ }_{2} \mathrm{O}$, providing evidence for the preferential formation of an TPPFe(III) $/$ TPPFe(III)-O-ion pair state instead of the desired TPPFe(II)/TPPFe(IV)=0.

Iron porphyrins serve as powerful photocatalysts and active site mimics of heme enzymes ${ }^{1,2}$ Bridged $\mu$-oxo iron porphyrins have been studied as potential atom-economical photocatalysts for oxygen atom transfer (OAT) in organic reactions. ${ }^{1-6}$ However, the quantum yields $(\Phi$, photons absorbed vs product formed) for these systems are poor $\left(\leq 10^{-4}\right) .^{4}$ This low efficiency has led to speculation about the excited state dynamics of these complexes. Based on picosecond optical transient absorption (OTA) of a simple bisporphyrin, $\mu$-oxo bis[tetraphenylporphyrinato iron(III)] ((TPPFe) ${ }_{2} \mathrm{O}$, Figure 1A), two main competing pathways have been proposed for the photochemistry of these systems after ligand-to-metal charge transfer (LMCT) excitation. In the first proposal (pathway $A$ in Figure $1 \mathrm{~B}$ ), one of the $\mathrm{Fe}-\mathrm{O}$ bonds of the $\mu$-oxo bridge undergoes a homolytic cleavage and separates into TPPFe(IV)=O and a bare TPPFe(II). ${ }^{4}$ The Fe(IV)-oxo subsequently reacts with a substrate to give the desired oxidized product. The original catalyst can then be reformed after the TPPFe(II) subunits react with $\mathrm{O}_{2}$, allowing for efficient substrate oxidation using a readily accessible O-atom source. If this pathway dominates the excited-state dynamics, the low quantum yield is likely caused by recombination of the TPPFe(II)/TPPFe(IV)=O subunits before substrate binding. Alternatively, the LMCT state could primarily disproportionate into a ligand-centred charge separated state composed of catalytically inactive TPPFe(III)+ and TPPFe(III)-O subunits (Figure 1B, Pathway $B$ ), with any small population of $\mathrm{Fe}(\mathrm{IV})=\mathrm{O}$ species below the detection threshold of transient absorption measurements. ${ }^{7}$ In this work, we use femtosecond

\footnotetext{
a. School of Chemical Sciences, University of Illinois at Urbana-Champaign, $600 \mathrm{~S}$. Mathews Ave., Urbana, Illinois 61801, USA.

E-mail: vuraweis@illinois.edu

†Authors contributed equally to this work. Electronic Supplementary Information (ESI) available: for methods, sample preparation, additional experimental details and data. See DOI: 10.1039/x0xx00000x
}
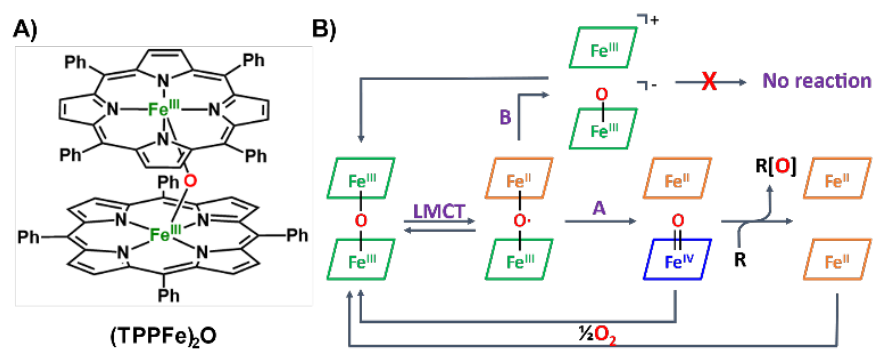

Figure 1 - (A) Structure of (TPPFe) ${ }_{2} \mathrm{O}$ (B) Proposed photocatalytic pathways in $\mu$-oxo iron bisporphyrins.

transient $\mathrm{M}_{2,3}$-edge spectroscopy and optical transient absorption to distinguish between these two pathways in the bisporphyrin (TPPFe) ${ }_{2} \mathrm{O}$, and identify the cause of its low quantum yield.

This dimer contains antiferromagnetically coupled high-spin $\mathrm{Fe}$ (III) porphyrins with a $\mu$-oxo bridge between the iron centres, separated by a distance of about $5.2 \AA . .^{8,9}$ Infrared, magnetic, and $\mathrm{X}$-ray crystallographic studies ${ }^{8}$ have shown that the iron centres in the stacked porphyrin have a structure akin to the high-spin iron in the monoporphyrin $\mathrm{Fe}\left(\right.$ III)TPPCI. (TPPFe) ${ }_{2} \mathrm{O}$ has been found to function as a photocatalyst for oxidation of triphenylphosphine ${ }^{10}$ and cyclic alkenes ${ }^{5}$ upon photoexcitation between 325 and $400 \mathrm{~nm}$. 4,6,7,11

To examine the early photodynamics of (TPPFe) ${ }_{2} \mathrm{O}$ and complement prior picosecond studies, femtosecond optical transient absorption (OTA) spectroscopy was performed on a degassed benzene solution of the stacked porphyrin at $400 \mathrm{~nm}$ (see SI for experimental details). Several key features are apparent in the transient spectra (Figure 2A): positive absorption features from 620-750 nm, 575-600 nm, and 460$525 \mathrm{~nm}$, and bleach features from 600-620 nm, 525-575 nm, and 460-440 $\mathrm{nm}$. The strong bleach feature at $560 \mathrm{~nm}$ and weaker bleach feature at $605 \mathrm{~nm}$ correspond to Q-band bleaches. The positive features centred at $515 \mathrm{~nm}$ and between $620 \mathrm{~nm}$ and $750 \mathrm{~nm}$ represent the formation of an excited state with new absorption features at these wavelengths. The bleach at $450 \mathrm{~nm}$ appears within the Soret band after $1 \mathrm{ps}$ and reaches its maximum intensity by 20 ps. The delayed formation of this bleach suggests an initial excited state species that lacks absorption in this region. Additionally, between $100 \mathrm{fs}$ and 2 ps, 

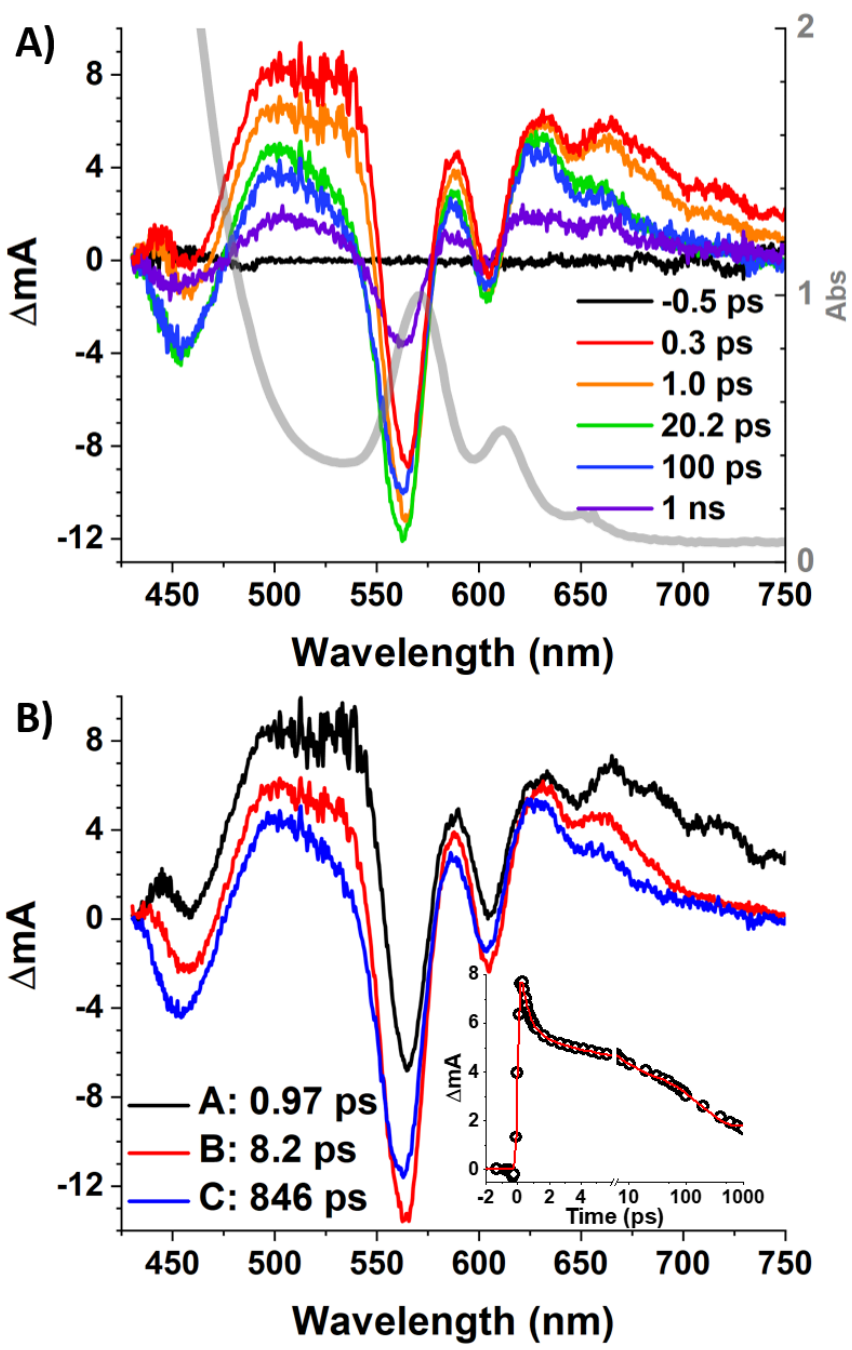

Figure 2 - (A) Optical transient absorption spectra of (TPPFe) ${ }_{2} \mathrm{O}$ (benzene) at select timepoints; grey trace is the static UV-Vis absorbance in benzene. (B) Spectral components from global fit. Inset: Kinetic slice at $515 \mathrm{~nm}$

the positive feature from $650 \mathrm{~nm}$ to $750 \mathrm{~nm}$ decays significantly and the $560 \mathrm{~nm}$ Q-band bleach feature increases intensity. Over the next $10-20 \mathrm{ps}$, absorption feature near $620 \mathrm{~nm}$ sharpens and the other features become slightly more negative. This spectrum then decays slowly over the next nanosecond, with significant intensity remaining at the end of the 1 ns time window. These long-lived features match well with prior OTA studies of (TPPFe) ${ }_{2}$ O. ${ }^{7,4}$

A global analysis of the transient spectra was performed using the program Glotaran. ${ }^{12} A$ three-state $A \rightarrow B \rightarrow C$ sequential model convolved with a Gaussian instrument response function (IRF) of $129 \mathrm{fs}$ was required to model the excited state spectra. Spectra of each component are shown in Figure 2B, with an inset showing a kinetic slice of the data and the fit at $515 \mathrm{~nm}$. Exponential lifetimes of $0.97 \pm 0.02 \mathrm{ps}, 8.2 \pm 0.3 \mathrm{ps}$, and $846 \pm 8$ ps for states A, B, and C, respectively, were extracted from the fit. The final lifetime is only approximate given the $1 \mathrm{~ns}$ time window of the experiment. As shown in the S.I. (Figures S2 through S5, Table S1), these dynamics do not change significantly upon excitation at 350 or $325 \mathrm{~nm}$, or in other

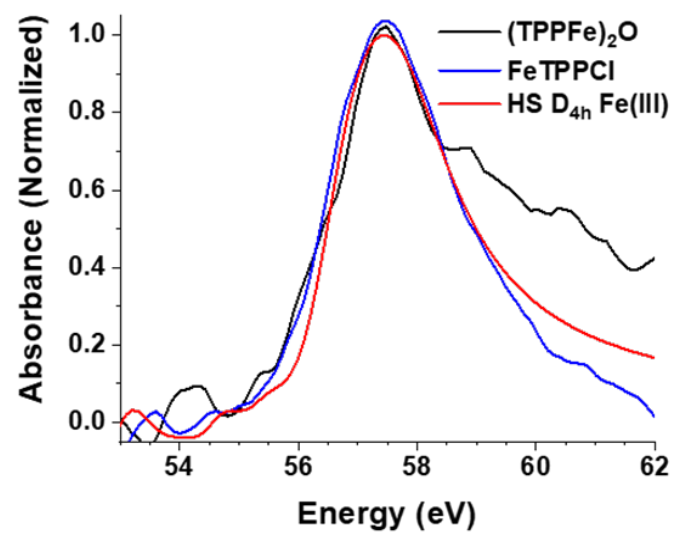

Figure 3 - Static $\mathrm{M}_{2,3}$-edge spectra of (TPPFe) ${ }_{2} \mathrm{O}$, FeTPPCl, and ligand field multiplet simulation of a high-spin $\mathrm{D}_{4 \mathrm{~h}}$ iron(III) centre.

solvents, suggesting that the excited states evolution is relatively insensitive to the experimental conditions.

Based on our prior work with FeTPPCl, ${ }^{13}$ we assign State A (not observed in earlier picosecond studies) as the LMCT excited state. The spectra of States B and C are similar to each other, but are markedly different from State A, featuring a bleach at $450 \mathrm{~nm}$ and lacking the broad absorption from 650-750 $\mathrm{nm}$. We therefore assign $B$ and $C$ as vibrationally hot and cold versions of the same electronic state. This state was previously assigned as either the ion pair or the $\mathrm{Fe}(\mathrm{II}) / \mathrm{Fe}(\mathrm{IV})$ state, a disagreement which we will resolve with transient XANES below.

To identify the short-lived electronic states of the $\mathrm{Fe}$ centres, we performed ultrafast transient $M_{2,3}$-edge $X$-ray absorption near-edge spectroscopy (XANES). $\mathrm{M}_{2,3}$-edge XANES measures the $3 p$-to-3d transition of first-row metals, and it provides comparable element, oxidation state, and ligand field specificity to L- and K-edge XANES. ${ }^{14}$ This technique was used to resolve the photophysics of several $3 d$ metal systems, 13,15,16 including Fe(III)TPPCl. The broadband XUV probe was generated using high-harmonic generation ( $\mathrm{HHG}$ ) employing a tabletop instrument described previously. ${ }^{14}$ For compatibility with the ultrahigh vacuum of the XUV spectrometer, the bisporphyrin was embedded in polystyrene film as a proxy for benzene solution. ${ }^{17}$ As shown Figure S6 in the S.I., the kinetics are nearly identical between solution and film.

The ground state XANES spectrum of (TPPFe) ${ }_{2} \mathrm{O}$ is shown in Figure 3. The bisporphyrin has a main peak centred at $57.4 \mathrm{eV}$ and is an excellent match to the spectrum of $\mathrm{Fe}(\mathrm{III}) \mathrm{TPPCl}$, supporting the assignment of each porphyrin as high-spin $\mathrm{Fe}(\mathrm{III})$. A ligand field multiplet simulation of high-spin square pyramidal Fe(III) is also shown, accurately predicts the single main peak and small pre-peaks from 54-56 eV. Transient $\mathrm{M}_{2,3}$ edge XANES was performed after $400 \mathrm{~nm}$ excitation, with an estimated excitation fraction of $8 \%$ per monomer. Figure $4 \mathrm{~A}$ contains the spectral slices (pump-on minus pump-off) from -0.27 ps (before time zero) to 9.0 ps. Time zero is independently measured by performing transient absorption of $\alpha-\mathrm{Fe}_{2} \mathrm{O}_{3}$. Upon excitation, a bleach feature centred at $57.4 \mathrm{eV}$ appears along with a positive feature centred at $55.0 \mathrm{eV}$. By 2 ps, these features decay to a weak positive signal at $56.0 \mathrm{eV}$. A global fit to an $A \rightarrow B$ model (with $B$ having an infinite lifetime on the timescale of this experiment) highlights these spectral 

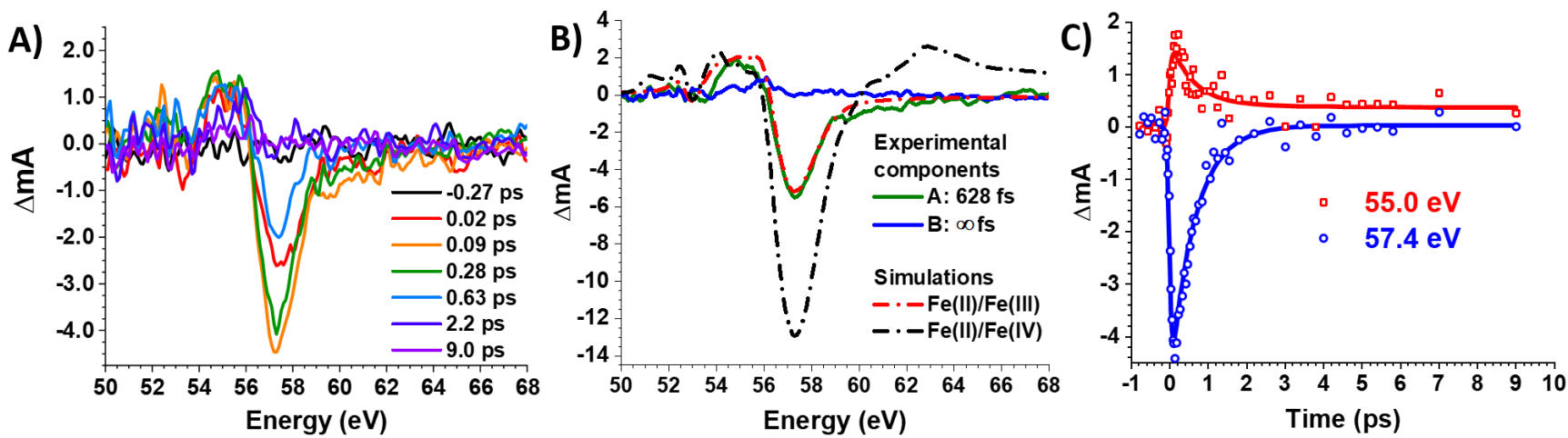

Figure 4 - Transient $\mathrm{M}_{2,3}$-edge XANES of (TPPFe) ${ }_{2} \mathrm{O}$ with $400 \mathrm{~nm}$ excitation: (A) Spectral slices at select time points. (B) Comparison the first spectral component with simulated $\mathrm{Fe}(\mathrm{II}) / \mathrm{Fe}(\mathrm{IV})$ and $\mathrm{Fe}(\mathrm{II}) / \mathrm{Fe}$ (III) spectra given an $8 \%$ excitation fraction.. (c) Kinetic traces at the $55.0 \mathrm{eV}$ excited state absorption and $57.4 \mathrm{eV}$ bleach, with global fit traces.

components, as shown in Figure 4B. Kinetic traces at 55.0 and $57.4 \mathrm{eV}$ are shown in Figure $4 \mathrm{C}$. Component $\mathrm{A}$ rises with an instrument response function (IRF) of $146 \pm 5$ fs FWHM and decays with an exponential time constant of $638 \pm 14 \mathrm{fs}$. This IRF is slightly longer than the $90 \mathrm{fs}$ response measured using $\alpha$ $\mathrm{Fe}_{2} \mathrm{O}_{3}$. Such a delayed rise was also observed in $\mathrm{Fe}(\mathrm{III}) \mathrm{TPPCl}$ and is caused by partial $\pi-\pi^{*}$ character in the initial excited state, which relaxes in tens of $\mathrm{fs}$ to a fully LMCT state. The $638 \mathrm{fs}$ decay of XUV state $A$ is a reasonably good match to the 1 ps decay of OTA state $A$.

In order to assign the transient XANES spectrum, ligand field multiplet simulations ${ }^{14,18,19}$ were performed for the potential $\mathrm{Fe}(\mathrm{II}) / \mathrm{Fe}$ (III) [LMCT] and $\mathrm{Fe}(\mathrm{II}) / \mathrm{Fe}(\mathrm{IV})=\mathrm{O}$ states. Note that in the TPPFe(III)+/TPPFe(III)-O- [ion pair] state, the irons' electronic structure has not changed significantly from the ground state, so minimal transient signal is expected in the metal-specific XANES probe. These simulations are shown in Figure $4 B$ and are scaled to the $8 \%$ excitation fraction. The LMCT state is predicted to have a ground state bleach at $57.4 \mathrm{eV}$ caused by loss of Fe(III) and excited state absorptions at 53 and $55 \mathrm{eV}$ caused by the formation of $\mathrm{Fe}(\mathrm{II})$. This simulation is a nearly quantitative match to the experimental spectrum of component $A$, both in magnitude and shape. The $\mathrm{Fe}(\mathrm{II}) / \mathrm{Fe}(\mathrm{IV})=\mathrm{O}$ state is calculated to have a ground-state bleach at $57.4 \mathrm{eV}$ about twice as large as that of the LMCT state because two Fe(III) signals are lost. The $\mathrm{Fe}(\mathrm{II})$ ion leads to positive absorption features at 53, 55 and the $\mathrm{Fe}(\mathrm{IV})$ causes a broad positive feature at $63 \mathrm{eV}$. The latter two peaks are not observed in the experimental spectrum, indicating that no $\mathrm{Fe}(\mathrm{IV})$ is formed with the signal-to-noise of the experiment. This weak positive signal in the long-lived state is consistent with ligand-centred excitation (either electronic or vibrational) that minimally perturbs the metal's d-orbital manifold. ${ }^{13}$

By combining the OTA and XTA results, a consistent picture emerges of the bisporphyrin excited state dynamics, which is summarized in Figure 5. Photoexcitation of the mixed $\pi$ $\pi^{*} / \mathrm{LMCT}$ band at $400 \mathrm{~nm}$ is followed by rapid relaxation into the $\mathrm{Fe}(\mathrm{II}) / \mathrm{Fe}$ (III) LMCT state (as shown by the delayed rise of the $\mathrm{Fe}(\mathrm{II})$ signal in XTA). This state decays to the TPPFe(III)+TPPFe(III)-O- ion pair in $\sim 1 \mathrm{ps}$, as shown by the loss of the Fe(II) signal in XTA and the change in shape of the OTA spectrum. After vibrational cooling, the ion pair state lasts for

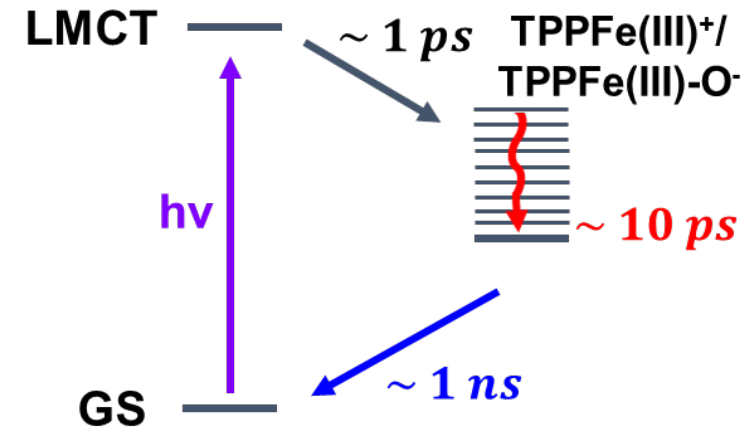

Figure 5 - Proposed dominant photodynamics for (TPPFe) ${ }_{2} \mathrm{O}$ approximately a nanosecond before recombining to the (TPPFe) ${ }_{2} \mathrm{O}$ ground state.

Overall, this picture shows that upon photoexcitation, the stacked porphyrin predominantly follows Pathway $B$ in Figure $1 \mathrm{~A}$ and not Pathway $A$. While the catalytic activity of $\mu$-oxo iron bisporphyrins suggests that the TPPFe(II)/TPPFe(IV)=O state is formed in at least small amounts, 4,20 the relative population compared to the ion pair state is low enough that it does not appear by the current experimental conditions. The disfavouring of the Fe(IV)-oxo state explains the low quantum yield of product formation but suggests a rational strategy for improving this yield. Synthetic modifications that favour the TPPFe(II)/TPPFe(IV)=O state, for example by destabilizing the ligand-centred ion pair, may shift the excited-state branching toward the catalytically active state and improve the overall quantum yield of these photocatalysts.

This material is based upon work supported by the U.S. Department of Energy, Office of Science, Office of Basic Energy Sciences under Award Number DE-SC0018904. This material is based upon work supported by the National Science Foundation Graduate Research Fellowship under Grant No. DGE-1746047. K. Sye thanks the Springborn Fellowship. We thank Alison Fout for insightful discussion and Yusef Shari'ati for help with global fitting.

\section{Conflicts of Interest}

There are no conflicts to declare. 


\section{Notes and references}

1 A. Maldotti, R. Amadelli, C. Bartocci, V. Carassiti, E. Polo and G. Varani, Coord. Chem. Rev., 1993, 125, 143-154.

2 J. Rosenthal, T. D. Luckett, J. M. Hodgkiss and D. G. Nocera, J. Am. Chem. Soc., 2006, 128, 6546-6547.

3 J. Rosenthal, B. J. Pistorio, L. L. Chng and D. G. Nocera, J. Org. Chem., 2005, 70, 1885-8.

$4 \quad$ M. W. Peterson, D. S. Rivers and R. M. Richman, J. Am. Chem. Soc., 1985, 107, 2907-2915. L. Weber, G. Haufe, D. Rehorek and H. Hennig, J. Chem. Soc. Chem. Commun., 1991, 502-503.

$6 \quad$ M. W. Peterson and R. M. Richman, 1985, 13, 722-725.

7 C. R. Guest, K. D. Straub, J. A. Hutchinson and P. M. Rentzepis, J. Am. Chem. Soc., 1988, 110, 5276-5280. A. B. Hoffman, D. M. Collins, V. W. Day, E. B. Fleischer, T. S. Snvastava and J. L. Hoard, J. Am. Chem. Soc., 1972, 94, 3620-3626.

E. B. Fleischer and T. S. Srivastava, J. Am. Chem. Soc., 1969, 91, 2403-2405.

R. M. Richman and M. W. Peterson, J. Am. Chem. Soc., 1982, 104, 5795-5796. K. S. Suslick, J. F. Bautista and R. A. Watson, J. Am. Chem. Soc., 1991, 113, 6111-6114.

12 J. J. Snellenburg, S. Laptenok, R. Seger, K. M. Mullen and I. H. M. Van Stokkum, J. Stat. Softw., 2012, 49, 1-22. E. S. Ryland, M.-F. Lin, M. A. Verkamp, K. Zhang, K. Benke, M. Carlson and J. Vura-Weis, J. Am. Chem. Soc., 2018, 140, 4691-4696.

14 K. Zhang, M. F. Lin, E. S. Ryland, M. A. Verkamp, K. Benke, F. M. F. De Groot, G. S. Girolami and J. Vura-Weis, J. Phys. Chem. Lett., 2016, 7, 3383-3387. K. Zhang, R. Ash, G. S. Girolami and J. Vura-Weis, J. Am. Chem. Soc., 2019, 141, 17180-17188.

16 S. Londo, S. Biswas, J. Husek, I. V. Pinchuk, M. J. Newburger, A. Boyadzhiev, A. H. Trout, D. W. McComb, R. Kawakami and L. R. Baker, J. Phys. Chem. C, 2020, 124, 11368-11375.

17 Y. Shari'ati and J. Vura-Weis, J. Synchrotron Radiat., 2021, 28, 1850-1857.

18 K. Zhang, G. S. Girolami and J. Vura-Weis, J. Synchrotron Radiat., 2018, 25, 1600-1608.

19 E. Stavitski and F. M. F. de Groot, Micron, 2010, 41, 687694.

20 P. K. Shantha and A. L. Verma, Inorg. Chem., 1996, 35, 2723-2725. 